\title{
Cardiac arrhythmias among hospitalized Coronavirus 2019 (COVID-19) patients: prevalence, characterization, and clinical algorithm to classify arrhythmic risk
}

\author{
Moshe Rav Acha ${ }^{1}$, Amir Orlev $^{1}$, Itay Itzhaki ${ }^{2}$, Shmuel F Zimmerman ${ }^{1}$, Bashar Fteiha ${ }^{1}$, \\ Davina Bohm ${ }^{1}$, Ramzi Kurd ${ }^{1}$, Tal Y Samuel ${ }^{1}$, Elad Asher ${ }^{1}$, Yigal Helviz ${ }^{1}$, Michael \\ Glikson $^{1}$, and Yoav Michowitz ${ }^{1}$ \\ ${ }^{1}$ Shaare Zedek Medical Center \\ ${ }^{2}$ Hebrew University Hadassah Medical School
}

September 21, 2020

\begin{abstract}
Objectives: A significant proportion of COVID-19 patients may have cardiac involvement including arrhythmias. Although arrhythmia characterization and possible predictors were previously reported, there are conflicting data regarding the exact prevalence of arrhythmias. Clinically applicable algorithms to classify COVID patients' arrhythmic risk are still lacking, and are the aim of our study. Methods: We describe a single center cohort of hospitalized patients with a positive nasopharyngeal swab for COVID-19 during the initial Israeli outbreak between $1 / 2 / 2020-30 / 5 / 2020$. The study's outcome was any documented arrhythmia during hospitalization, based on daily physical examination, routine ECG's, periodic 24-hour Holter, and continuous monitoring. Multivariate analysis was used to find predictors for new arrhythmias and create classification trees for discriminating patients with high and low arrhythmic risk. Results: Out of 390 COVID-19 patients included, 28 (7.2\%) had documented arrhythmias during hospitalization, including: 23 atrial tachyarrhythmias, combined atrial fibrillation (AF) and ventricular fibrillation, ventricular tachycardia storm, and 3 bradyarrhythmias. Only $7 / 28$ patients had previous arrhythmias. Our study showed significant correlation between disease severity and arrhythmia prevalence $(\mathrm{p}<0.001)$ with a low arrhythmic prevalence among mild disease patients (2\%). Multivariate analysis revealed background heart failure (CHF) and disease severity are independently associated with overall arrhythmia while age, CHF, disease severity, and arrhythmic symptoms are associated with tachyarrhythmias. A novel decision tree using age, disease severity, CHF, and troponin levels was created to stratify patients into high and low risk for developing arrhythmia. Conclusions: Dominant arrhythmia among COVID-19 patients is AF. Arrhythmia prevalence is dependent on age, disease severity, CHF, and troponin levels. A novel simple Classification tree, based on these parameters, can discriminate between high and low arrhythmic risk patients.
\end{abstract}

\section{WHAT'S KNOWN?}

- A significant proportion of COVID-19 patients may have cardiac involvement including arrhythmias.

- There is a correlation between disease severity in general and cardiac involvement specifically to occurrence of cardiac arrhythmias.

- Arrhythmia characterization and possible predictors.

\section{WHAT'S NEW?}

- Using a 24-hour Holter monitoring among hospitalized COVID-19 patients, for better arrythmias detection.

- Among of all hospitalized COVID-19 patients, $7.2 \%$ had new arrhythmias during hospitalization.

- Classification tree which discriminate between high and low arrhythmic risk patients 


\section{Introduction}

During the last year the world has faced a new Corona virus pandemic, named COVID-19. Although COVID-19 clinical manifestations are mainly respiratory, cardiac involvement exists in a substantial number of patients, and cardiac arrhythmias have been reported in variable percent of patients, ranging from $6 \%$ to $19 \%$ of overall hospitalized COVID patients and from $9 \%$ to $44 \%$ of COVID patients hospitalized in intensive care units (ICU). ${ }^{1,2,3}$ In some patients, arrhythmias represent the first clinical manifestation of COVID infection, ${ }^{4,5}$ while in others arrhythmia may occur only during the recovery out-of-hospital period. ${ }^{6}$ Overall, there seems to be a correlation between disease severity in general and cardiac involvement specifically to occurrence of cardiac arrhythmias, ${ }^{2,4,6}$ although sudden cardiac death was described among mild COVID19 patients as well. ${ }^{4}$ Moreover, a significant minority of severe COVID ICU patients with arrhythmias had normal troponin levels, suggesting other mechanisms rather than cardiac injury, triggering arrhythmias in these patients. ${ }^{3,7}$ Various potential causes for cardiac involvement and arrhythmias were suggested, including: a) direct viral myocardial injury- myocarditis, ${ }^{2,4,6}$ b) cytokine-induced myocardial injury as well as cytokine-mediated conduction disturbances ${ }^{8,9}$ and QT prolongation $;^{3}$ c) hypoxemia, d) hypotension, e) hyper-adrenergic state, f) drug effect, such as hydroxychloroquine or azithromycin, which can lead to prolonged QT-related Torsades de Pointes as well as to nodal and infra-nodal conduction disturbances. ${ }^{4,10,11}$ Although much data has accumulated regarding various cardiac complications in COVID-19, the characterization and exact prevalence of cardiac arrhythmias among COVID-19 patients and more so among the mild-moderate ones is still unclear. ${ }^{4,6}$ Furthermore, although various possible predictors for cardiac arrhythmias in COVID patients were speculated, ${ }^{2,4,6,7,9}$ a simple clinical algorithm to classify arrhythmic risk has not been developed so far. Such algorithm is critical to guide cardiac monitoring level during the hospitalization period and maybe during the recovery period as well. The primary aim of our study was to characterize the various arrhythmias encountered among hospitalized COVID-19 patients of various severity levels, describe their prevalence and develop a clinical algorithm to classify arrhythmic risk.

\section{Methods}

\section{Study configuration and patients}

Retrospective analysis of a single center cohort of hospitalized COVID-19 patients. A computer-based search of all patients who were hospitalized in Shaare Zedek medical center in Jerusalem during the period of $1 / 2 / 2020-30 / 5 / 2020$ (including those admitted to regular corona departments as well as those admitted or transferred to corona intensive care unit) with nasopharyngeal swab PCR positive for severe acute respiratory syndrome-coronavirus 2 (SARS-Cov -2), who were at the age of [?]18 years old at the day of admission. Notably, the research period represents the period of the first COVID-19 outbreak in Israel.

\section{Study outcomes}

The study primary outcome was any arrhythmia documented during the hospitalization. The search for arrhythmias was based on review of all patients' medical records, 12-lead ECG's, 24-hour Holters or continuous ECG monitoring (telemetry).

Our secondary aim was to evaluate possible predictors for arrhythmia occurrence, with regard to:

1. Patients' medical history; including age, gender, hypertension (HTN), diabetes mellitus (DM), ischemic heart disease (IHD), background congestive heart failure (CHF), and previous documented arrhythmia

2. Presenting "arrhythmic" symptom as palpitations or syncope

3. Disease severity. Disease severity was categorized primarily according to the patients' respiratory status, and categorized as mild for those who did not need any $\mathrm{O}_{2}$ support during their hospitalization; moderate for those who required some $\mathrm{O}_{2}$ support via nasal cannula or mask; severe for those who required non-invasive ventilation (high-flow, CPAP, etc); and critical for those who needed mechanical ventilation or alternatively those with multi-organ failure who were supported by inotropes. Notably, disease severity was determined according to the patients' worst respiratory or hemodynamic status during their hospitalization period. 
4. Presence of cardiac injury as assessed by high-sensitive cardiac troponin-I (hsTnI) and/or Trans Thoracic Echocardiography (TTE).

5. Inflammatory status based on D-DIMER and CRP lab measurements

6. QTc duration, as evidenced by review of all patients ECG's performed during the hospitalization period, determining the ECG with longest QTc duration. Notably, to look for other potential contributors for prolonged QTc we documented the patient's electrolytes and medications around the day of longest QTc ECG. The QTc was calculated using the Bazett formula. In patients with heart rate of less than 60 QT and not QTc was measured.

7. COVID-19 treatments, especially regarding use of Hydroxychloroquine and Azithromycin and other QT-prolonging medications

\section{ECG, Holter monitoring, and laboratory data}

- Physical examination including checking for heart regularity and rate was done on daily basis. - Routine ECG was performed on admission and whenever new cardiac symptoms appeared or an arrhythmia was suspected on physical examination or telemetry. - Laboratory examination was not done routinely but on clinical basis for patients with cardiac symptoms, general deterioration and before treatment with Hydroxychloroquine and/or Azithromycin. . - 24-hour Holter monitoring was performed in a minority of patients, primarily those with evidence of cardiac injury, abnormal ECG on admission, or those with significant illness.

\section{Statistical analysis}

Categorical variables summarized as frequency and percentage. Continuous variables were evaluated for normal distribution using histograms and Q-Q plots and reported as median and interquartile range. Independent samples T-test and Mann-Whitney test were applied to compare between patients with and without new arrhythmia. Chi-square test and Fisher's exact test were used to compare categorical variables. Multi variate Logistic regression using forward stepwise (likelihood ratio) selection method ( $\mathrm{p}<0.05$ was used as criteria for inclusion) was used to explore variables associated with new arrhythmia. Variables available for inclusion were: age, gender, BMI, history of HTN, DM, IHD, CHF, valvular heart disease, lung disease, presentation with syncope or palpitations, past arrhythmia, and COVID-19 illness severity. Classification trees were applied to identify subgroup of patients that are in increased risk for arrhythmia. Classification and Regression Tree (CART) and Chi-square Automatic Interaction Detection (CHAID) growing algorithms were used. The following variables were available for the classification trees: age, gender, previous history of HTN, DM, IHD, CHF, valvular heart disease, lung disease, presentation with syncope or palpitations, past arrhythmia, and COVID-19 illness severity. A second model included also hsTnI and CRP levels. All statistical tests were 2 sided and $\mathrm{p}<0.05$ was considered statistically significant. SPSS software was used for all statistical analyses (IBM SPSS statistics for windows, version 24, IBM cooperation, Armonk, NY, USA, 2016).

The study was approved by the hospital ethical committee.

\section{Results}

\section{Study population}

After exclusion of 6 patients whose age was less than 18 years old, the study included 390 patients with confirmed COVID-19 infection. Median patients age was 57.5 years (IQR 43-74.3 years), the prevalence of HTN, DM, ischemic heart disease (IHD) and CHF was 30\%, 20.3\%, 8.7\% and 6.4\%, respectively. Twenty-six patients had a past history of arrhythmias: 2 with bradyarrhythmias due to a previous atrioventricular (AV) block with an implanted pacemaker for both; and 24 with tachyarrhythmias including atrial fibrillation (AF) in 21 (4 persistent and 17 paroxysmal), ventricular fibrillation in 1 patient [(patient with dilated cardiomyopathy implanted with an implantable cardioverter-defibrillator (ICD)], supraventricular tachycardia ( SVT) in 1 patient and atypical atrial flutter in 1 patient. On presentation, most patients had respiratory symptoms $(67.7 \%)$ while a minority presented also with either palpitations or syncope $(4.4 \%)$. Thirty-eight $(9.7 \%)$ patients were hospitalized in intensive care unit (including 8 with new arrhythmias). Relevant blood 
test, ECG findings and distribution of disease severity are further presented in Table 1. Twenty-nine (7.4\%) patients died during the index hospitalization.

\section{New arrhythmias}

Of the study cohort, 28/390 patients $(7.2 \%)$ presented or developed new arrhythmias during hospitalization (Table 2). Seven of these 28 patients $(25 \%)$ had previous documented arrhythmias. There were 3 cases of bradyarrhythmias and 25 tachyarrhythmias. The first bradyarrhythmic patient was a 92 -year-old woman who presented with complete AV block associated with a QT of $550 \mathrm{~ms}$ and normal electrolytes; the second case was a 51-year-old man who presented with 2:1 AV block with left bundle branch block (LBBB) and developed complete AVB within hours from his admission. Notably, the patient had narrow complex QRS on a clinic visit two years earlier. Both patients did not receive any negative dromotropic drugs; laboratory examinations revealed normal electrolytes, mildly elevated hsTnI (100 and $83 \mathrm{ng} / \mathrm{L}$, respectively; normal values for men women $<35 \mathrm{ng} / \mathrm{L}$ and $20 \mathrm{ng} / \mathrm{L}$, respectively) and elevated CRP (15 and $4.5 \mathrm{mg} / \mathrm{dl}$, respectively; normal value $<0.5 \mathrm{mg} / \mathrm{dL}$ ). Both had pacemakers implanted during their index hospitalization. A third patient was a 33 year-old man who presented in sinus rhythm without any conduction abnormality and who developed on day 2 transient sinus bradycardia with slow ventricular escape rhythm that resolved within a few hours (Figures $1 \mathrm{~A}$ and $1 \mathrm{~B}$ ). Notably, the patient's $\mathrm{O}_{2}$ saturation at that time was $84 \%$ and later rose to $90 \%$, his electrolytes were normal and no drugs with negative chronotropic effects were given. Laboratory examinations revealed elevated CRP of $16.58 \mathrm{mg} / \mathrm{dl}$ and normal hsTnI $(10 \mathrm{ng} / \mathrm{L})$.

There were 25 patients with new tachyarrhythmias: 24 of them had new atrial tachyarrhythmias including 20 with paroxysmal AF. Of the 24 patients with atrial tachyarrhythmias, 7 had a prior history of similar arrhythmias. Notably, during his index hospitalization, one of these patients with new-onset AF also had a documented ventricular fibrillation which was successfully cardioverted (Table 2). This patient did not have prolonged QT and his electrolytes were normal; however, he did have elevated hsTnI of $7049 \mathrm{ng} / \mathrm{L}$ and mildly elevated CRP $(6.8 \mathrm{mg} / \mathrm{dl})$. Three patients had atrial flutter and 1 patient had an SVT episode (narrow complex regular tachycardia of $195 \mathrm{bpm}$ seen on monitor and terminated with a Valsalva maneuver). Lastly, there was a patient with ventricular tachycardia (VT) storm (Table 1). This patient had an ischemic cardiomyopathy for which he was previously implanted with an ICD. He presented with normal sinus rhythm, however, a week later had respiratory deterioration necessitating mechanical ventilation. He was transferred to ICU where he developed ventricular bigeminy (Figures $1 \mathrm{C}$ and $1 \mathrm{D}$ ) which deteriorated within an hour to recurrent monomorphic VT episodes. The patient received multiple ICD therapies and his VT's eventually resolved after intravenous lidocaine and amiodarone. Notably, the patient did not receive ionotropic medications, his QTc was stable around $480 \mathrm{~ms}$ and electrolytes were normal, hsTnI increased to $17,578 \mathrm{ng} / \mathrm{L}$ and his maximal $\mathrm{CRP}$ was $16.58 \mathrm{mg} / \mathrm{dL}$. Importantly, none of the new arrhythmias were discovered by Holter.

\section{Comparison of patients with and without new arrhythmia (overall arrhythmias)}

Comparison of the 28 patients with new arrhythmias with those who did not experience any arrhythmia is presented in Table 1. Patients with new arrhythmia were older and had more comorbidities as DM, HTN, IHD and valvular heart disease. Also, their disease was more significant, with $28.6 \%$ and $17.9 \%$ of patients categorized as critical or severe disease in the new arrhythmia group compared with $7.2 \%$ and $8.8 \%$ in the non arrhythmia group, respectively $(\mathrm{p}<0.001)$. Among the new arrhythmia group there were more patients presenting with respiratory symptoms and lower proportion of patients who had mild illness $(14.3 \%$ versus $55 \%, \mathrm{p}<0.001)$. The new arrhythmia group patients were more likely to receive a QT prolonging drug, mostly an antiarrhythmic agent, while there was no difference regarding percent of patients on "anti-COVID" medications as Hydroxychloroquine and/or Azithromycin. On ECG, the QRS width and QTc were longer in patients with new arrhythmias and the prevalence of bundle branch block was higher. Laboratory tests of patients with arrhythmia also reflected a more severe illness, with higher creatinine, troponin, CRP and DDimer levels. Noteworthy, there was a significantly increased mortality among the arrhythmia group $(32.1 \%$ versus $5.5 \%, \mathrm{p}<0.001)$. 


\section{Comparison of patients with and without new tachyarrhythmias}

Comparison of patients with new tachyarrhythmias with the no arrhythmias group (Supplementry Table 1) shows that patients with new tachyarrhythmias $(n=25,6.4 \%)$ were older, had more comorbidities, and experienced a more severe disease. Rate of respiratory symptoms was higher among patients with new tachyarrhythmias, however it was not statistically significant $(\mathrm{p}=0.07)$. As expected, more patients with new tachyarrhythmias received a QT prolonging drug which included mainly anti-arrhythmic medications. Differences in other ECG and laboratory results are further described in Supplementary table 1.

\section{Multivariate Logistic regression model for new arrhythmia and new tachyarrhythmias.}

Multivariate logistic regression models were conducted to explore variables that were associated with new arrhythmias and new tachyarrhythmias. As presented in Figure 2, background CHF (OR 4.66, 95 CI 1.4514.97, $\mathrm{p}=0.01)$ and disease severity $(\mathrm{p}=0.003)$ were associated with overall new arrhythmias. A stepwise increase in odds ratio (OR) for overall new onset arrhythmia was seen with increased disease severity. As compared with mild disease, patients with moderate, severe and critical disease had OR of 4.81, 8.86 and 15.79 to develop new arrhythmia, respectively.

Factors associated with new tachyarrhythmias are presented in Figure 3. Age, background CHF, presentation with syncope or palpitations and disease severity were significantly associated with new tachyarrhythmia in the multivariate analysis.

\section{Classification trees.}

Classification trees were applied in order to identify subgroup of patients who are at low or high risk for arrhythmia. Figure 4 demonstrates a classification tree incorporating baseline patients' characteristics including prior arrhythmias, as well as clinical symptoms and disease severity. As seen, the first division for discriminating patients with and without new arrhythmia is based on age. Patients aged [?] 70 compared to $<70$ years had $18.1 \%$ vs. $2.6 \%$ prevalence of arrhythmias, respectively. The next divisions of the model include illness severity and background CHF. The highest prevalence of arrhythmias was $33.3 \%$ seen in patients aged [?] 70 years and critical disease severity. The lowest prevalence of $1.6 \%$ was seen among patients aged $<70$ years with mild to moderate disease severity. A second classification tree incorporated the same clinical parameters of the first tree with addition of hsTnI levels and CRP (Figure 5). Of note, hsTnI levels were measured in 219/390 patients (56\%). As seen in Figure 5, hsTnI was the best discriminator for the new arrhythmia occurrence and obviated the contribution of other clinical parameters. Patients with negative or not measured hsTnI had an arrhythmia prevalence of $2.1 \%$ compared to patients with troponin [?] $48 \mathrm{ng} / \mathrm{L}$ who had an arrhythmia prevalence of $34.1 \%$.

\section{Discussion}

Our study including a single center cohort of 390 COVID-19 patients revealed 28/390 (7.2\%) cases with documented cardiac arrhythmias, including 20 cases of AF, 3 atrial flutter, 1 VF (in a patient who also had paroxysmal AF), 1 VT case and 3 bradyarrhythmic cases. The study showed significant correlation between disease severity and arrhythmia prevalence, revealing significant increase in arrhythmia prevalence with increasing disease severity [11/116 (9.5\%); $5 / 37$ (13.5\%); 8/34 (23.5\%) for moderate, severe, and critical severity, respectively, $\mathrm{p}<0.001$ ] with a very low prevalence of arrhythmias among patients with mild COVID19 disease $(4 / 203 ; 2 \%)$. Multivariate forward regression analysis showed that background CHF and disease severity are independently associated with overall arrhythmias while age, background CHF, disease severity, and arrhythmic symptoms (syncope or palpitations) are associated with tachy-arrhythmias. Importantly, a classification tree using specific age (>70 years old) and troponin ([?] $48 \mathrm{ng} / \mathrm{L}$ ) cutoffs, as well as disease severity, and history of CHF could further stratify patients into high and low risk for developing arrhythmia. To our knowledge, these relevant cutoffs were not previously described and our presented classification tree is novel in this regard.

The overall arrhythmia prevalence in our study was $7.2 \%$, which is lower than reported in some studies ${ }^{1,12}$ and similar to others. ${ }^{13,14}$ Notably, the initial study from China reported an overall prevalence of $16.7 \%$ 
among 138 hospitalized COVID-19 patients in Wuhan, ${ }^{1}$ but there was a significant difference of arrhythmia incidence between non-ICU and ICU patients $(6.3 \%$, vs. 44.4\%; p 0.001) and their relatively high overall prevalence was clearly influenced by the high number of ICU patients $(36 / 138,26 \%)$ in whom there was very high prevalence of arrhythmias. Similarly, among 115 American COVID-19 patients, ${ }^{12}$ a dramatic change in arrhythmia prevalence was noticed between non-ICU and ICU patients ( $0 \%$ versus $27.5 \%$, p 0.0002$)$, with an overall arrhythmia prevalence of $16.5 \%$ given the fact that $69 / 115$ study patients $(60 \%)$ were ICU patients. ${ }^{12}$ In contrast, an Italian study focusing on 132 stable non-ICU COVID-19 patients, reported 12/132 (9\%) new arrhythmias documented during the admission period. ${ }^{13}$ Our overall arrhythmic prevalence is similar to Wuhan's non-ICU group and to the Italian stable non-ICU patients, probably reflecting the low percentage of ICU patients $(9.7 \%)$ in our cohort. Notably, the arrhythmic prevalence among our ICU patients was significantly greater than our non-ICU patients (21\% versus $5.7 \%$; 0.003$)$, in accordance with the previous studies. Similar findings were reported in a single center study including 700 patients (11\% ICU patients), revealing an overall arrhythmia prevalence of $7.5 \%$ with marked difference of arrhythmia prevalence between ICU and non-ICU patients. ${ }^{14}$ Lastly, the low arrhythmic prevalence among the non-ICU group compared with the ICU one, suggests that need for mechanical ventilation or presence of multi-organ dysfunction necessitating ionotropic support, which characterized our "critical" severity and most of our ICU patients, have major impact on arrhythmic prevalence as was suggested by previous studies. ${ }^{1,6,9,12.14}$

$\mathrm{AF}$ or atrial flutter was the dominant arrhythmia in our study, occurring for $23 / 28$ (82\%) of cases with documented arrhythmia, while only a minority of these cases had previous arrhythmias $(5 / 23,22 \%)$. This result is in line with multiple previous studies, revealing AF to be the most prevalent arrhythmia in COVID19 disease. ${ }^{1,6,12,13,14,15}$ The absence of previous atrial arrhythmias in most of these patients and the fact that some of these new arrhythmia was timely correlated with respiratory deterioration, may suggest an association or perhaps even a causative relation between COVID illness and arrhythmia development. Indeed, some have suggested that the increased cytokine levels among severe COVID patients may trigger atrial arrhythmias. ${ }^{7,8}$ Nevertheless, one should not interpret the above results as a definite cause and effect relation between COVID disease and AF, as most of these patients are elderly with multiple comorbidities as HTN, diabetes and IHD and most if not all have significant respiratory symptoms, all of which are well known risk factors for AF occurrence. Thus, we like others, ${ }^{13,14}$ find it hard to confirm a necessary pathophysiologic link between AF occurrence and COVID-19 infection. Regardless of whether AF is a direct result of COVID or circumstantial to the multiple comorbidities of these patients, multiple publications suggest AF to predict bad prognosis among COVID-19 patients. ${ }^{8,12,14,16}$

There were 3 cases with new onset bradyarrhythmias in our study, including two cases with advanced AVB and one with a transient slow ventricular escape rhythm. Notably, 2 of these cases were relatively young male patients (50 and 33 year old) without prior conduction abnormalities who presented with advanced AVB or developed a slow ventricular escape rhythm, respectively. Both did not receive any negative dromotropic or chronotropic drug. Both had normal electrolytes and markedly elevated inflammatory markers with mildly increased or normal hsTnI. Accordingly, we raise the possibility, which we cannot prove, that these bradyarrhythmias had been related to the cytokine storm and exacerbated inflammatory state associated with COVID-19 illness. Interestingly, inflammatory-mediated conduction abnormalities have been suggested in previous reports of COVID-19 patients who developed otherwise unexplained AVB during the acute COVID illness, with markedly elevated inflammatory markers. ${ }^{8,9}$

Disease severity, categorized by the level of respiratory support needed, was the most robust clinical predictor for new-onset arrhythmia among COVID-19 patients in our study. This correlation was shown in most previous studies, with special attention to the remarkably high arrhythmia prevalence among COVID-19 ICU patients, many of whom were mechanically ventilated and/or with multi-organ failure necessitating inotropic support. ${ }^{1,2,3,12,14}$ The other clinical predictor for new-onset overall arrhythmias was background CHF, which is well-established risk factor for atrial and ventricular arrhythmias. ${ }^{17,18}$

Regarding laboratory predictors, previous studied suggested both myocardial injury as well as inflammatory process to have impact on arrhythmic prevalence or even underlie arrhythmia development, ${ }^{7,12}$ with the 
notion of "inflammatory and cardiac injury mediated atrial tachycardia and conduction disturbances". ${ }^{7,8,9}$ Indeed, in our study both hsTnI and CRP were significantly associated with new arrhythmias in the univariate analysis. However, in the classification tree model, hsTnI per se was found to best differentiate between low and high arrhythmic activities (Fig 5). This is in line with many previous studies suggesting myocardial injury, assessed via troponin levels, to correlate with need for mechanical ventilation, ICU admission, mortality and arrhythmias. ${ }^{1,2,3,4,9,19}$

Importantly, our classification trees (Fig 4\&5) are noval and clinically relevant as they can discriminate between patients with high and low risk for new onset arrhythmia. Although, some of these variables as age and troponin are clinically intuitive and were previously reported,,$^{2,4,9,14}$ no cutoffs were previously given to guide clinical decisions. Accordingly, we suggest that patients whose age is below 70 with mild to moderate disease ( $1.6 \%$ arrhythmic prevalence) or with negative hsTnI ( $2.1 \%$ arrhythmic prevalence) may be hospitalized to a "non-monitored bed" and even discharged early; while patients aged [?]70 (18.1\% arrhythmic prevalence) or patients with hsTnI [?] $48 \mathrm{ng} / \mathrm{L}$ (34.1\% arrhythmic prevalence) should be carefully monitored for the occurrence of arrhythmias. These algorithms might be of crucial importance to direct optimal utilization of medical staff and monitoring equipment in COVID-19 outbreaks, especially in areas with limited medical resources.

\section{Conclusion}

Arrhythmias, mostly AF episodes, may be seen in $7.2 \%$ of hospitalized patients with COVID-19 disease. Baseline clinical factors including age [?] 70, disease severity and hsTnI levels may separate between patients with high and low probability for arrhythmias and thus dictate further level of patient monitoring during hospitalization, or on the other hand in the low risk patients, the ability for early patient discharge.

\section{Limitations}

The main limitation of our study is its retrospective nature. Nevertheless, given the unexpected nature of this pandemia, prospective study would not be realistic. Other limitations includes the fact that not all hospitalized COVID-19 patients underwent laboratory measurements of troponin and/or CRP, thus, having a built-in bias for patients with cardiac symptoms or higher disease severity levels. Moreover, since only part of our patients underwent a 24-hour Holter and continuous ECG monitoring, some transient arrhythmias might have been missed on examination and 12-lead ECG's. Lastly, we do not have post-discharge follow-up

data; thus, our study has probable some underestimation of true COVID-related arrhythmia prevalence, as some arrhythmia could probably develop post discharge.

\section{References}

1. Wang, D, Hu, B, Hu, C, Zhu, F, Liu, X, Zhang, J, Wang, B, Xiang, H, Cheng, Z, Xiong, Y, Zhao, Y, Li, Y, Wang, X, \& Peng, Z. Clinical Characteristics of 138 Hospitalized Patients with 2019 Novel Coronavirus-Infected Pneumonia in Wuhan, China. JAMA. 2020; 323:1061-1069.

2. Shi S, Qin M, Shen B, Cai Y, Liu T, Yang F, Gong W, Liu X, Liang J, Zhao Q, Huang H, Yang B, Huang C. Association of cardiac injury with mortality in hospitalized patients with COVID-19 in Wuhan, China. JAMA Cardiology 2020; 5(7) : 802-810.

3. Driggin E, Madhavan MV, Bikdeli B, Chuich T, Laracy J, Biondi-Zoccai G, Brown TS, Der Nigoghossian C, Zidar DA, Haythe J, Brodie D, Beckman JA, Kirtane AJ, Stone GW, Krumholz HM, Parikh SA. Cardiovascular considerations for patients, health care workers, and health systems during the COVID-19 pandemic. J Am Coll Cardiol 2020; 18s: 735-801.

4. Kochi AN, Tagliari AP, Forleo GB, Fassini GM, Tondo C. Cardiac and arrhythmic complications in patients with COVID-19. J Cardiovasc Electrophysiol 2020; 31: 1003-1008.

5. Hu H, Ma F, Fang Y. Coronavirus fulminant myocarditis saved with glucocorticoid and human immunoglobulin. Eur Heart J 2020; https://doi.org/10.1093/eurheartj/ehaa190.

6. Lakkireddy DR, Chung MK, Deering TF, Gopinathannair R, Albert CM, Epstein LM, Harding CV, Hurwitz JL, Jeffery CC, Krahn AD, Kusumoto FM, Lampert R, Mansour M, Natale A, Patton KK, Seiler A, Shah MJ, Wang PJ, Russo AM. Guidance for rebooting electrophysiology through the COVID- 
19 pandemic from the Heart Rhythm Society and the American Heart Association Electrocardiography and Arrhythmias Committee of the Council on Clinical Cardiology: Endorsed by the American College of Cardiology. Heart Rhythm. 2020 Jun 12:S1547-5271(20)30554-3

7. Lazzerini PE, Boutjdir M, Capecchi PL. COVID-19 arrhythmic risk and inflammation: mind the gap. Circulation 2020; 142: 7-9.

8. Kochav SM, Coromilas E, Nalbandian A, Ranard LS, Gupta A, Chung MK, Gopinathannair R, Biviano AB, Garan H, Wan EY. Cardiac arrhythmias in COVID-19 infection. Circ Arrhythm Electrophysiol 2020;13(6).

9. Guo T, Fan Y, Chen M, Wu X, Zhang L, He T, Wang H, Wan J, Wang X, Lu Z. Cardiovascular implications of fatal outcomes of patients with coronavirus disease. JAMA Cardiol 2020. 5(7) :1-8.

10. Verny C, de Gennes C, Sébastien P, Lê Thi HD, Chapelon C, Piette JC, Chomette G, Godeau P. Heart Conduction Disorders in Long-Term Treatment with Chloroquine. Two New Cases. Press Med. 1992; 21: 800-4.

11. Capel RA, Herring N, Kalla M, Yavari A, Mirams GR, Douglas G, Bub G, Channon K, Paterson DJ, Terrar DA, Burton RA. Hydroxychloroquine reduces heart rate by modulating the hyperpolarizationactivated current If: novel electrophysiological insights and therapeutic potential. Heart Rhythm 2015; 12: 2186-2194.

12. Colon CM, barrios JG, Chiles JW, McElwee SK, Russel DW, Maddox WR, Kay GN. Atrial arrhythmias in COVID-19 patients. JACC Clinical electrophysiology 2020. Epublished DOI:10.1016/j.jacep.2020.05.015.

13. Sala S, Peretto G, De Luca G, Farina N, Campochiaro C, Tresoldi M, Dagna L, Zangrillo A, Gulletta S, Della Bella P. Low prevalence of arrhythmias in clinically stable COVID-19 patients. Pacing Clin Electrophysiol 2020; https://doi.org/10.1111/pace.13987

14. Bhatla A, Mayer MM, Adusumalli S, Hyman MC, Oh E, Tierney A, Moss J, Chahal AA, Anesi G, Denduluri S, Domenico CM, Arkles J, Abella BS, Bullinga JR, Callans DJ, Dixit S, Epstein AE, Frankel DS, Garcia FC, Kumareswaram R, Nazarian S, Riley MP, Santangeli P, Schaller RD, Supple GE, Lin D, Marchlinski F, Deo R. COVID-19 and cardiac arrhythmias. Heart Rhythm 2020; 22 : S1547-5271.

15. Gopinathannair R, Merchant FM, Lakkireddy DR, Etheridge SP, Feigofsky S, Han JK, Kabra R, Natale A, Poe S, Saha SA, Russo AM. COVID-19 and cardiac arrhythmias: a global perspective on arrhythmia characteristics and management strategies. J Interventional Cardiac Electrophysiol 2020; $3: 1-8$.

16. Onder G, Rezza G, Brusaferro S. Case-fatality and characteristics of patients dying in relation to COVID-19 in Italy. JAMA 2020; 323: 1775-1776.

17. Ponikowski P, Voors AA, Anker SD, Bueno H, Cleland JGF, Coats AJS, Falk V, González-Juanatey JR, Harjola VP, Jankowska EA, Jessup M, Linde C, Nihoyannopoulos P, Parissis JT, Pieske B, Riley JP, Rosano GMC, Ruilope LM, Ruschitzka F, Rutten FH, van der Meer P. 2016 ESC Guidelines for the diagnosis and treatment of acute and chronic heart failure: The Task Force for the diagnosis and treatment of acute and chronic heart failure of the European Society of Cardiology (ESC)Developed with the special contribution of the Heart Failure Association (HFA) of the ESC. Eur Heart J 2016; 37:2129.

18. Lip GY, Heinzel FR, Gaita F, Juanatey JR, Le Heuzey JY, Potpara T, Svendsen JH, Vos MA, Anker SD, Coats AJ, Haverkamp W, Manolis AS, Chung MK, Sanders P, Pieske B, Gorenek B, Lane D, Boriani G, Linde C, Hindricks G, Tsutsui H, Homma S, Brownstein S, Nielsen JC, Lainscak M, Crespo-Leiro M, Piepoli M, Seferovic P, Savelieva I. European Heart Rhythm Association/Heart Failure Association joint consensus document on arrhythmias in heart failure, endorsed by the Heart Rhythm Society and the Asia Pacific Heart Rhythm Society. Europace 2016; 18: 12-36.

19. Chapman AR, Bularga A, Mills NL. High-Sensitivity Cardiac Troponin Can Be an Ally in the Fight Against COVID-19. Circulation 2020.

Figure legends

Figure 1: ECG example of 2 patients with new arrhythmias . 
(A) and (B) ECG of a 33-year-old patient. On 8:22 the ECG shows normal sinus rhythm with heart rate of 90 beats per minute (bpm) (A). Ninty minutes later while awake and with low $\mathrm{O}_{2}$ saturation he developed extreme sinus bradycardia with ventricular escape (B). ECG done a few hours later (not shown) demonstrated return of sinus at $90 \mathrm{bpm}$. (C) ECG during sinus rhythm of $78 \mathrm{bpm}$ showing RBBB of a patient who later exhibited VT storm. (D) ECG a day later showed sinus rhythm and ventricular bigeminy. Few hours later the patient developed VT storm. (see text for further discussion on both patients).

Figure 2: Multi variate Logistic regression analysis for the occurrence of new arrhythmia. History of CHF and disease severity in a step wise fashion were independently associated with the occurrence of new arrhythmias. $\mathrm{OR}=$ odds ratio.

Figure 3: Multi variate Logistic regression analysis for the occurrence of new tachyarrhythmia. Age, history of CHF, symptoms of palpitations or syncope and disease severity were independently associated with the occurrence of new tachyarrhythmias. $\mathrm{OR}=$ odds ratio.

Figure 4: Classification tree for new arrhythmia based on clinical parameters. Age and disease severity level were found to discriminate best new arrhythmia prevalence. Age [?] 70 was associated with $18.1 \%$ prevalence of new arrhythmia, while age $<70$ in patients with mild-moderate disease was associated with low $(1.6 \%)$ prevalence. $\mathrm{NL}=$ normal, no arrhythmia; $\mathrm{NA}=$ new arrhythmia; $\mathrm{CHF}=$ congestive heart failure.

Figure 5: Classification tree for new arrhythmia based on clinical and laboratory parameters. This classification tree incorporated the same clinical parameters as in first tree with the addition of hsTnl and $\mathrm{CRP}$ as potential variables. In this model HsTnl (Normal $<35 \mathrm{ng} / \mathrm{L}$ ) was found as the best discriminator for arrhythmia prevalence; as HsTnl [?] $48 \mathrm{ng} / \mathrm{L}$ was associated with $34.1 \%$ prevalence of new arrhythmia, while in patients with negative or not-measured hsTnl the prevalence was $2.1 \%$. HsTnl= high sensitivity troponin-I; NA= new arrhythmia.

\section{Hosted file}

Tables.docx available at https://authorea.com/users/360522/articles/482187-cardiacarrhythmias-among-hospitalized-coronavirus-2019-covid-19-patients-prevalencecharacterization-and-clinical-algorithm-to-classify-arrhythmic-risk

\section{Hosted file}

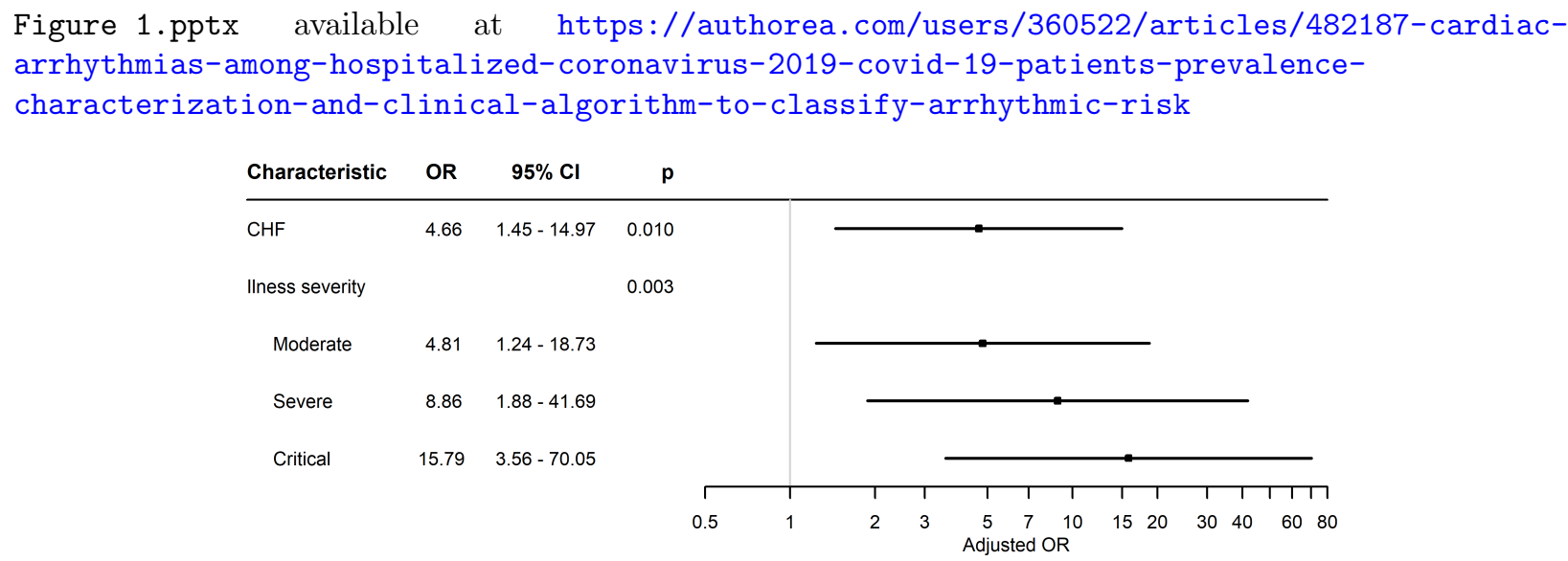




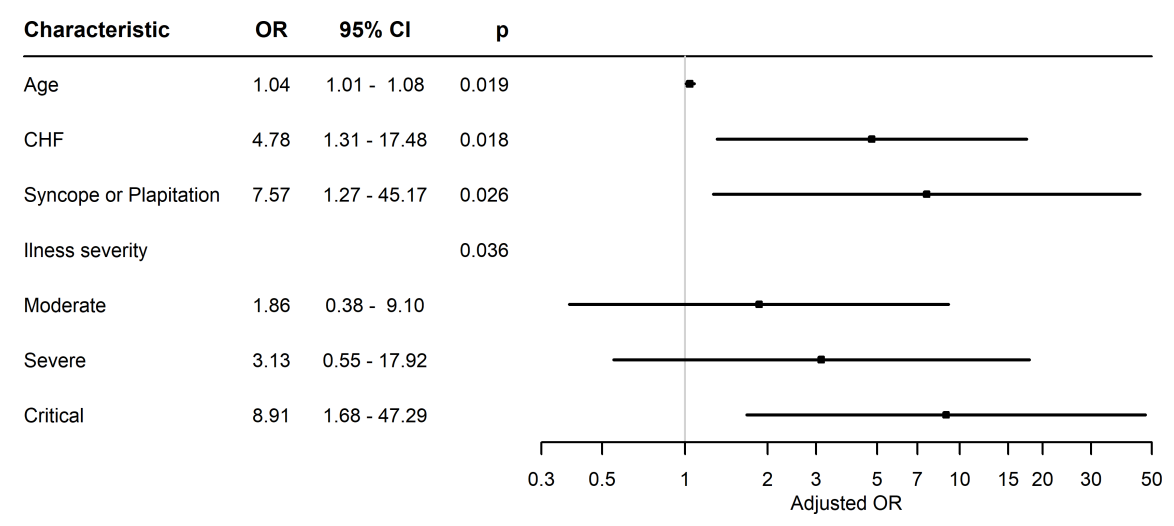

\section{Hosted file}

figure 4.pptx available at https://authorea.com/users/360522/articles/482187-cardiacarrhythmias-among-hospitalized-coronavirus-2019-covid-19-patients-prevalencecharacterization-and-clinical-algorithm-to-classify-arrhythmic-risk

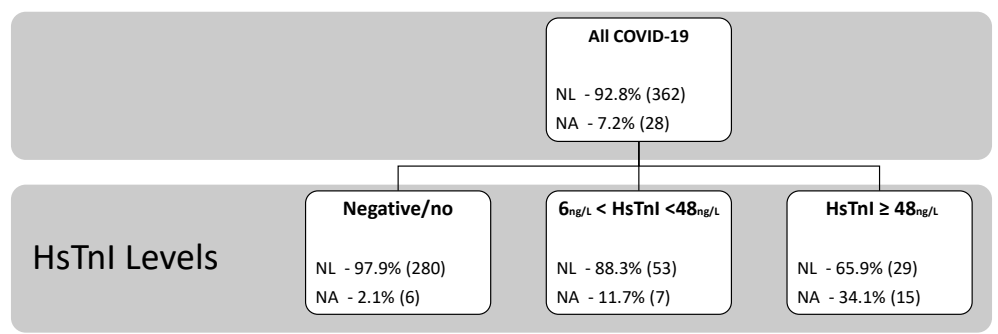

